\title{
Bronchial response pattern of antigen presenting cells and regulatory $T$ cells in children less than 2 years of age
}

\author{
I Heier, ${ }^{1}$ K Malmström, ${ }^{2}$ A S Pelkonen, ${ }^{2}$ L P Malmberg, ${ }^{2}$ M Kajosaari, ${ }^{3}$ M Turpeinen, ${ }^{2}$ \\ H Lindahl, ${ }^{3}$ P Brandtzaeg, ${ }^{1} \mathrm{~F}$ L Jahnsen, ${ }^{1}$ M J Mäkelä ${ }^{2}$
}

${ }^{1}$ LIIPAT, the Pathology Clinic, University of Oslo,

Rikshospitalet-Radiumhospitalet Medical Centre, Oslo, Norway;

${ }^{2}$ Division of Allergy, Department of Medicine Helsinki University Central Hospital, Helsinki, Finland; ${ }^{3}$ Hospital for Children and Adolescents, Helsinki University Central Hospital, Helsinki, Finland

Correspondence to: Dr F L Jahnsen, Institute of Pathology, Rikshospitalet, N-0027 Oslo, Norway:

f.l.jahnsen@medisin.uio.no

FLJ and MJM share senior authorship.

Received 18 April 2007 Accepted 16 January 2008

Published Online First

4 February 2008

\begin{abstract}
Background: In early childhood, the ability to mount protective immune responses in the airways is impaired, with increased risk of allergic sensitisation to inhaled allergens. Antigen presenting cells (APC) and regulatory T cells (Treg) are important modifiers of $T$ cell immunity but little is known about their distribution in bronchial mucosa at this age. Here the subset distribution of APC and the appearance of Foxp3 ${ }^{+}$Treg and bronchus associated lymphoid tissue (BALT) were examined immunohistochemically in children less than 2 years of age with chronic asthma-like symptoms of the lower airways.
\end{abstract}

Methods: Immunophenotyping was performed in situ on bronchial biopsy specimens obtained from 45 infants, 4-23 months of age, under investigation for airway disease.

Results: A well developed HLA-DR ${ }^{+}$network of APC was present in all samples, approximately $50 \%$ of the cells being $\mathrm{CD}^{2} 8^{+}$macrophages and the remainder various subsets of dendritic cells. The density of $\mathrm{HLA}^{-\mathrm{DR}^{+}}$cells increased significantly with age but was not related to atopy, clinical symptoms or lung function. Comparing the density of APC subsets and clinical parameters, only the number of intraepithelial CD1 $\mathrm{a}^{+}$dendritic cells was significantly increased in infants who had recently suffered a respiratory infection. BALT structures were identified in 22 children, with no relation to lung function, atopic status or human rhinovirus positivity. Plasmacytoid dendritic cells and Foxp3 ${ }^{+}$Treg were located primarily within these isolated lymphoid follicles.

Conclusion: A bronchial network of dendritic cells and macrophages develops quite rapidly after birth, apparently independent of clinical symptoms or atopy. The high frequency of BALT structures containing putative tolerogenic dendritic cells and Treg suggests that these lymphoid follicles play an important role in bronchial immune homeostasis during infancy.

Several lines of evidence suggest that delayed postnatal maturation of $\mathrm{T}$ cell mediated immune functions increases the risk of allergic sensitisation and airway infections which are both important causes of childhood morbidity. ${ }^{12}$ One characteristic of the fetal and infant $T$ cell system is their polarisation towards Th2 cytokine dominated responses with low capacity for interferon $\gamma$ production. ${ }^{3}{ }^{4}$ This apparent deficiency in Th cell function is not necessarily an inherent cellular characteristic but can be ascribed to immaturity within subsets of antigen presenting cells (APC) that regulate $\mathrm{T}$ cell activity. Dendritic cells (DC), the primary professional APC, have a unique capacity to activate naïve $\mathrm{T}$ cells and polarise $\mathrm{T}$ cell differentiation. ${ }^{5}$ It is believed that neonatal DC display reduced antigen presenting capacity and impaired ability to deliver Th1 polarising signals to naive $\mathrm{T}$ cells, thus contributing to a deviation towards Th2 polarised immunity in infancy. ${ }^{46}$ However, this information is mainly derived from studies of peripheral blood derived DC.

In both adult humans and rodents, airway APC constitute a dense network strategically located beneath and within the conducting airway epithelium. ${ }^{78}$ In experimental animal models, mucosal airway DC efficiently capture and transport inhaled antigens to regional lymph nodes where they modify local T cell activity. ${ }^{9}$ Information on infant airways is limited but studies performed on autopsy material have suggested that the number of mucosal APC is low at birth and increases only slowly during the first year of life. Low numbers and delayed maturation of airway DC have also been demonstrated in infant rats, most likely contributing to defective regional immunity. ${ }^{10}$ Deficient $T$ cell function in infancy therefore appears to be derived both from a low local number of APC and their functional immaturity.

In humans, two functionally distinct DC precursor subsets, myeloid (m) DC and plasmacytoid (p) DC, occur in peripheral blood. Although both subsets differentiate into efficient APC after maturation, activated $\mathrm{mDC}$ and $\mathrm{pDC}$ display intrinsic differences and drive distinct types of $\mathrm{T}$ cell responses. Recently it was shown that matured pDCs have a unique ability to generate interleukin 10 producing regulatory $\mathrm{T}$ cells (Treg), both from the memory ${ }^{11}$ and naïve ${ }^{12} \mathrm{~T}$ cell population; in the latter case, depending on selective expression of ICOS ligand on pDCs. The relative densities of blood $\mathrm{pDC}$ and $\mathrm{mDC}$ differ between infants and adults, with pDC being more frequent in cord blood and $\mathrm{mDC}$ dominating in adult peripheral blood. ${ }^{13}$ Also, adult human airway mucosa contains mainly $\mathrm{mDC},{ }^{78}$ but whether the situation is different in early life is currently unknown.

In addition to DC, Treg determine the immunological outcome by suppressing $\mathrm{T}$ cell effector function, with the potential of limiting inflammation and immunopathology. ${ }^{14}$ Impaired function of Treg has been linked to various immune disorders, including autoimmunity, asthma and allergy. ${ }^{15} 16$ Studies have shown that $\mathrm{CD} 4^{+} \mathrm{CD} 25^{+}$Foxp3 ${ }^{+}$Treg develop in early fetal life and exist in numbers equivalent to adults in cord blood. ${ }^{17}$ However, whether these cells occur in the respiratory tract during infancy has not been determined. 
Table 1 Patient characteristics

\begin{tabular}{lcccc}
\hline & Group A & Group B & Group C & Total \\
\hline No of patients & 14 & 18 & 13 & 45 \\
Age (months) (median (range)) & $9.3(5.0-23.3)$ & $12.5(5.0-18.7)$ & $9.4(4.0-21.6)$ & $9.6(4.0-23.3)$ \\
Duration of symptoms (\% of age) (median (range)) & $55(18-96)$ & $57(28-99)$ & $78(19-100)$ & $59.6(18.7-100)$ \\
Sex (No of females) (n (\%)) & $6(43)$ & $5(28)$ & $4(31)$ & $15(33)$ \\
Atopy (n (\%)) & $11(79)$ & $8(44)$ & $8(62)$ & $27(60)$ \\
Symptoms and findings (n (\%)) & $10(71)$ & $12(67)$ & $9(69)$ & $31(69)$ \\
$\quad$ Wheeze ever & $6(43)$ & $8(44)$ & $5(39)$ & $19(42)$ \\
Wheeze frequently & $8(57)$ & $14(78)$ & $9(69)$ & $31(69)$ \\
Cough ever & $6(43)$ & $8(44)$ & $5(39)$ & $19(42)$ \\
Wheeze and/or cough between colds & $5(36)$ & $4(22)$ & $3(23)$ & $12(27)$ \\
Dyspnoea & $4(29)$ & $8(44)$ & $1(8)$ & $13(29)$ \\
Respiratory infection in previous 6 weeks & $8 / 13(62)$ & $9 / 15(60)$ & $2 / 12(17)$ & $19 / 40(48)$ \\
Positive for human rhinovirus on PCR* &
\end{tabular}

Group $A$, reversible airway obstruction; group $B$, irreversible airway obstruction; group $C$, normal lung function.

*Data not available for all patients.

Here, we characterised in situ the phenotypic distribution of resident APC as well as Foxp3 ${ }^{+}$Treg in the bronchial mucosa of clinically well characterised children, between 4 and 23 months of age, with early symptoms of asthma-like airway disease.

\section{MATERIAL AND METHODS \\ Subjects}

The patients $(n=61)$ included in the study have previously been described in detail. ${ }^{18}$ For immunohistochemistry, 16 biopsy samples were excluded because of inadequate size or tissue quality, while 45 were found to be acceptable (table 1). All patients had been referred to the Skin and Allergy Hospital and the Hospital for Children and Adolescents, Helsinki University Central Hospital, for investigation of persistent respiratory symptoms, including dyspnoea, coughing and wheezing. They had been symptomatic for at least 4 weeks within the preceding 2 months. As part of the clinical workup, the patients underwent allergy testing, lung function tests by whole body plethysmography and rigid bronchoscopy. Atopy was defined by a positive skin prick test to food or aeroallergens and/or the presence of atopic dermatitis.

The patients were divided into three groups according to their specific airway conductance (sGaw): group A, decreased sGaw with bronchodilator reversibility; group B, decreased sGaw without bronchodilator reversibility; and group C, normal sGaw. Reversibility was defined as a $30 \%$ increase in sGaw in response to inhalation of the $\beta_{2}$ agonist salbutamol (see Saglani and colleagues ${ }^{18}$ for details). Most patients underwent bronchoscopy within 3 weeks of plethysmography. None had been treated with steroids, either locally or systemically, for the last 6 weeks prior to bronchoscopy.

Bronchoscopy was clinically indicated whereas biopsy was performed for research purposes. Rigid bronchoscopy was performed in the hands of experienced operators, being as safe as flexible bronchoscopy, ${ }^{19}$ and giving a better view that allowed harvesting of somewhat larger biopsies with adequate structural preservation.

Written informed consent from parents was obtained and the study was approved by the local ethics committee. There were no complications associated with the endoscopic procedures.

\section{Biopsy material and immunostaining}

Bronchial biopsy specimens obtained from the main carina were formalin fixed and paraffin embedded, and serial sections were cut at $4 \mu \mathrm{m}$. For optimal staining results heat induced epitope retrieval was performed by boiling the sections in a microwave oven for $20 \mathrm{~min}$ in citrate buffer ( $\mathrm{pH}$ 6.0). Immunoenzyme staining on dewaxed tissue sections was performed in a Ventana NexEs IHC instrument (Tucson, Arizona, USA) with the standardised iView $\mathrm{DAB}$ or enhanced V-Red (alkaline phosphatase) detection kits, as recommended by the manufacturer. The primary monoclonal antibodies (mAbs) were directed against human HLA-DR (clone TAL.1B5, IgG1; DakoCytomation, Glostrup, Denmark), CD68 (clone PG-M1, IgG3; DakoCytomation), CD1a (clone MTB1, IgG1; Novocastra, UK), CD123 (a mixture of clone 7G3, IgG2a and clone 9F5, IgG1; BD Pharmingen, California, USA) and CD20 (clone L26, IgG2a; DakoCytomation). A polyclonal rabbit antihuman CD3 (dilution 1/50; DakoCytomation) was additionally applied. To identify Treg, we applied two different mAbs directed against Foxp3 in parallel staining experiments. Antihuman CD3 was combined with either rat mAb to Foxp3 (clone PCH101, IgG2a; Nordic Biosite, Sweden) or mouse antihuman Foxp3 mAb (clone 259D/C7, IgG1; BD Pharmingen), followed by Alexa Fluor 488 goat antirabbit IgG (Molecular Probes, Eugene, Oregon, USA) and Cy3 conjugated donkey antirat IgG (Jackson ImmunoResearch, Pennsylvania, USA) or Cy3 conjugated goat antimouse IgG (Jackson Immunoresearch). Appropriate isotype and concentration matched control reagents ensured immunostaining specificity.

Stained tissue sections were examined blindly by the same investigator $(\mathrm{IH})$ at $\times 400$ magnification. The cell density in the epithelium was recorded as the total number of positive cell profiles per basement membrane length unit $(1 \mathrm{~mm})$, whereas the cell number in lamina propria was recorded as positive cell profiles $/ \mathrm{mm}^{2}$. The total number of positive mucosal cells was recorded per basement membrane length unit by adding the intraepithelial counts and the lamina propria counts to a depth of the grid. On average, 18 visual fields were counted in each specimen (range 4-72). The mucosal areas containing lymphoid aggregates (see results section) were omitted from the cell enumeration.

\section{Human rhinovirus diagnostics}

PCR on bronchial specimens was performed on 40 of these 45 patients described here. The procedure is described in detail elsewhere. $^{20}$

\section{Statistics}

Non-parametric tests were applied for statistical analysis. For analysis of between group differences, the Kruskal-Wallis (three 
Figure 1 Formalin fixed and paraffin embedded bronchial mucosa from six different children (aged 4-23 months). Immunoenzymatic staining for (A, B) HLADR; (C) CD3 (brown colour) and CD20 (red); and (D) CD123. Paired immunofluorescence staining for $(E, F)$ CD3 (green) and Foxp3 (clone PCH101; red). (A) HLA-DR ${ }^{+}$cells occur both in the epithelium and lamina propria. Note cellular extensions reaching the luminal surface (arrows). (B-D) Approximately half of the specimens contained organised structures of bronchus associated lymphoid tissue (BALT) identified as cell aggregates containing $\mathrm{HLA}-\mathrm{DR}^{+}$cells $(\mathrm{B})$, $T$ and $B$ cells $(C)$, as well as $C D 123^{+}$cells with plasmacytoid morphology (D). Regulatory $T$ cells identified as $\mathrm{CD}^{+}{ }^{+} \mathrm{Foxp}^{+}$cells were located mainly in the BALT structures (E) and only in some sections scattered in the lamina propria $(F)$. Asterisk (F) indicates epithelium. Scale bars: $(A, B, D, E, F) \times 400$ magnification; $(C) \times 600$ magnification.
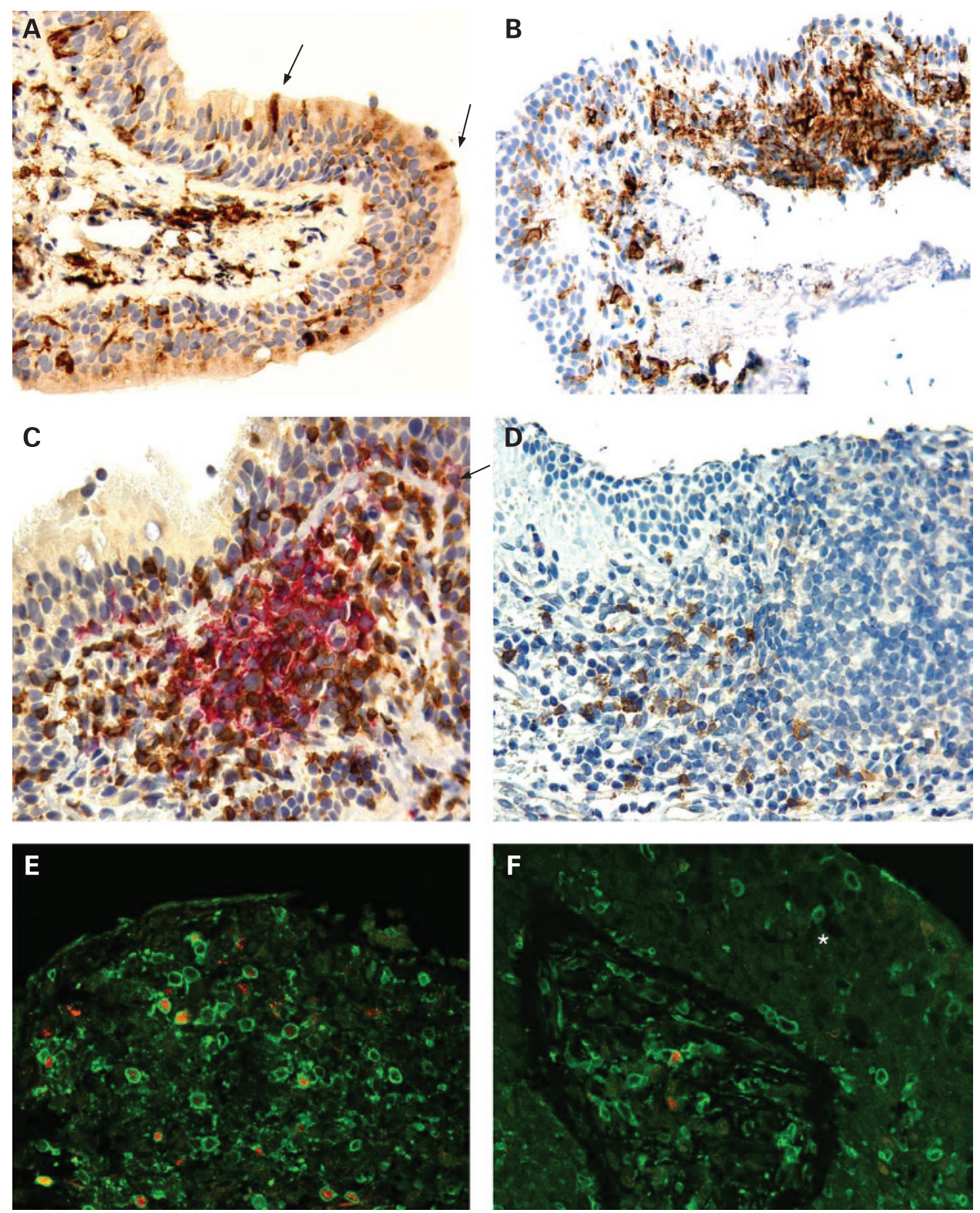

groups) and Mann-Whitney (two groups) tests were applied. For correlations, the Spearman's rank order coefficient was used. SPSS 12.0.1 for windows (Chicago, Illinois, USA) and GraphPad Prism 4 (San Diego, California, USA) were used for the statistical analyses.

\section{RESULTS}

\section{Antigen presenting cells}

A network of putative APC, identified as cells expressing the pan-APC marker HLA-DR, was detected both within the surface epithelium and in the subepithelial part of the lamina propria in all specimens examined $(n=45)$. The HLA-DR ${ }^{+}$cells displayed variable morphology, from rounded small cells to larger cells with long dendrites (fig 1A). Interestingly, in many of the samples we observed that intraepithelial $\mathrm{HLA}-\mathrm{DR}^{+}$cells extended projections between epithelial cells reaching into the airway lumen (fig 1A, arrows). These "snorkelling" APC constituted less than $5 \%$ of all intraepithelial APC, but a more accurate estimation of the density was difficult to obtain as the surface epithelium was variably preserved. The mucosal APC were evenly distributed but in almost half of the specimens isolated subepithelial aggregates of $\mathrm{HLA}-\mathrm{DR}^{+}$cells intermingled with $\mathrm{B}$ and $\mathrm{T}$ cells were observed, as described in more detail below (fig 1B, 1C).

When comparing the three patient groups, no significant differences in the numbers of APC were detected within the surface epithelium (fig 2A) or in the lamina propria (fig 2B). This was also the case for the total number of mucosal APC (group A: median $82 / \mathrm{mm}$ basement membrane, interquartile range (IOR) 57 to 114; group B: median 96 (IOR 63 to 138); and group C: median 136 (IOR 69 to 142))

When the patients were considered together, the atopic and non-atopic subjects appeared to be similar with regard to total numbers of APC (fig 3A, B). Also, within each separate patient group, there was no significant difference in total mucosal number of HLA-DR ${ }^{+}$cells between those deemed to be atopic or non-atopic (data not shown). The total number of HLA-DR ${ }^{+}$ cells increased significantly with age $(r=0.41, p=0.005$; fig 4$)$, although being quite variable, with some of the highest counts found in the youngest patients. 

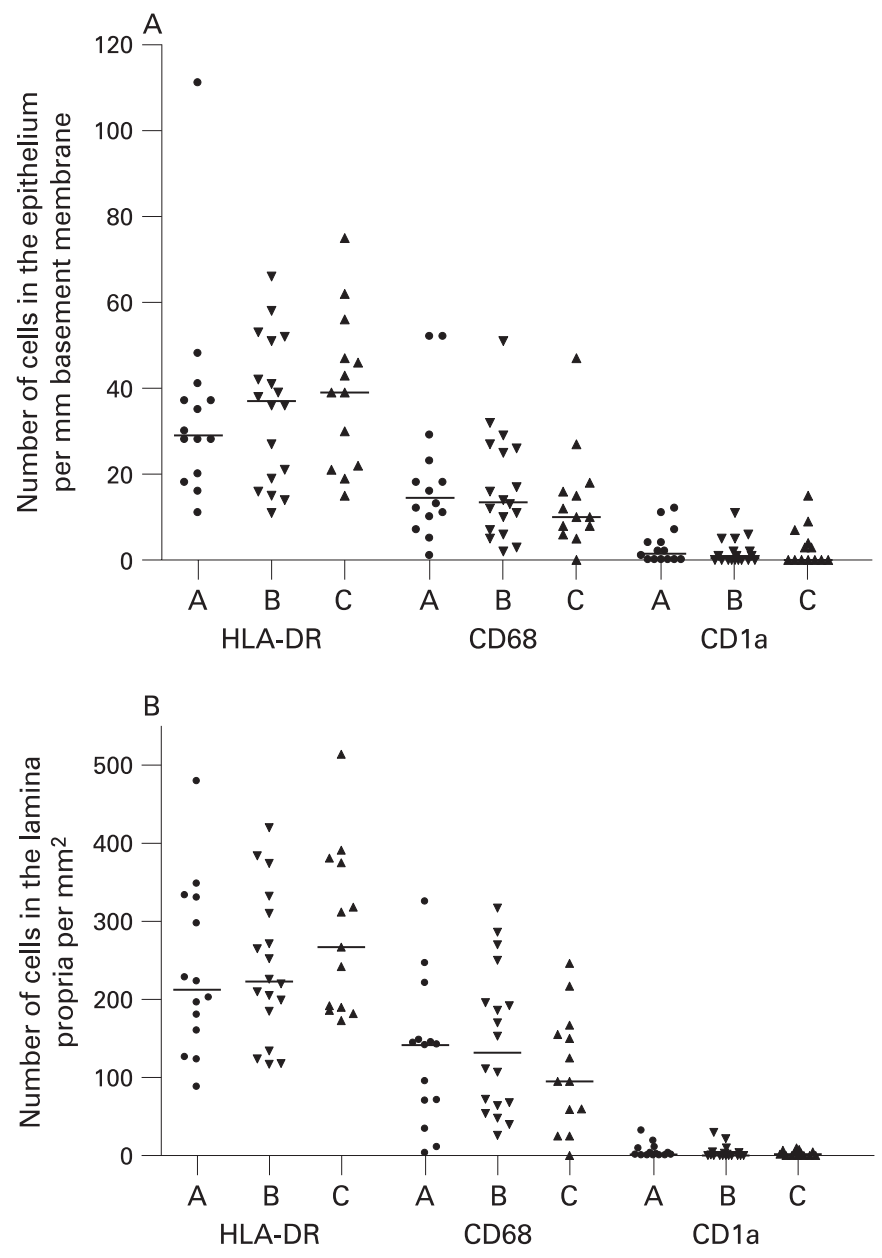

Figure 2 Numbers of cells expressing HLA-DR, CD68 or CD1a in the epithelium (A) and lamina propria (B) of bronchial mucosa from patients having impaired lung function with bronchodilator reversibility (group A), impaired lung function without bronchodilator reversibility (group B) or normal lung function (group C). Horizontal bars represent medians. There were no significant differences in cell numbers with any of the markers between the groups (Kruskal-Wallis test).

We and others have previously shown that most HLA-DR ${ }^{+}$ cells in human airway mucosa are either macrophages or different subtypes of DC. ${ }^{721}$ To further phenotype the mucosal APC population, we applied mAbs to various DC and macrophage markers. Macrophages, identified by the restricted isoform of CD68, constituted approximately $50 \%$ of the mucosal APC, both within the surface epithelium and in the lamina propria (fig 2). CD1a, a marker that identifies a subset of airway DC, was almost exclusively expressed by cells in the epithelial compartment (fig 2). The pDC marker CD123 was expressed on many cells within subepithelial cellular aggregates (fig 1D; see below), but only very few CD123+ cells were found in the lamina propria outside these isolated lymphoid follicles. CD123 is not an unequivocal marker for pDC, as it is also expressed on basophils. However, in this material, CD123+ cells clearly showed a distinct plasmacytoid morphology suggesting that they indeed were pDC (fig 1D).

The frequency and distribution of $\mathrm{CD}^{+} 8^{+}$macrophages, $\mathrm{CD} \mathrm{a}^{+} \mathrm{DC}$ and $\mathrm{pDC}$ were quite similar to what we have observed previously in the nasal mucosa of healthy adults. ${ }^{7}$ In the upper airway mucosa of adults, we found that most DC expressed CD1c, a marker present on most myeloid CD11 $c^{+}$DC
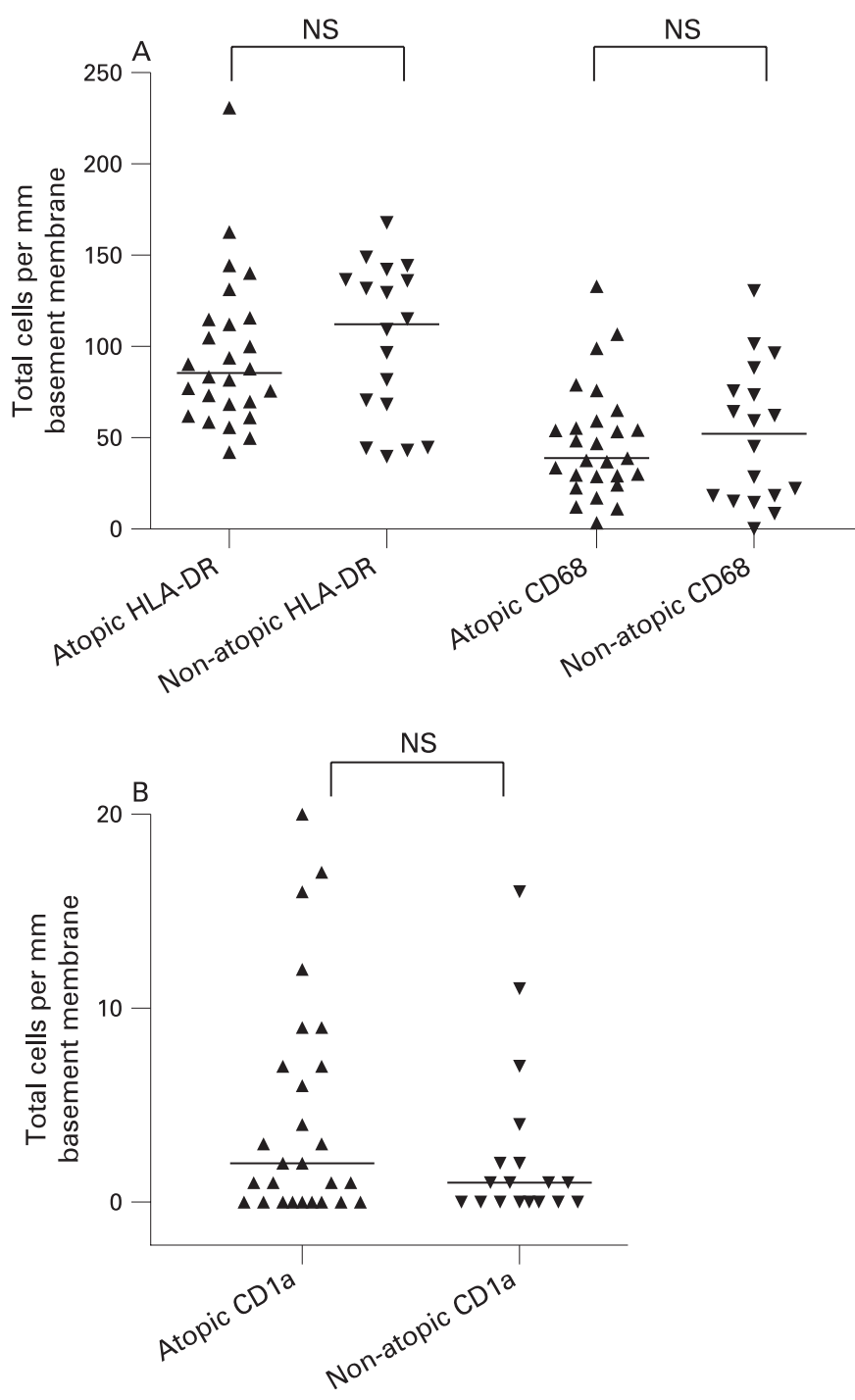

Figure 3 Numbers of cells expressing HLA-DR or CD68 in patients with or without atopy (A). Numbers of cells expressing CD1a or CD123 in the same clinical groups (B). Cell numbers are expressed as stained cells/ $\mathrm{mm}$ basement membrane, adding the number of intraepithelial cells and the number of lamina propria cells to a depth of $0.25 \mathrm{~mm}$. Horizontal bars represent medians. There were no significant differences in cell numbers between groups (Mann-Whitney test).

precursors in peripheral blood. ${ }^{7}$ Unfortunately, we were unable to achieve satisfactory staining with $\mathrm{mAbs}$ to CD1c on formalin fixed tissue sections even after various demasking procedures, so we could not confirm that this marker was also expressed on APC in the present study.

Human rhinovirus (HRV) was detected in $47.5 \%$ of the patients tested; primarily those with abnormal lung function (table 1 and Lindahl and colleagues ${ }^{19}$ ). The numbers of CD68 ${ }^{+}$ macrophages were higher in individuals who were HRV positive compared with those who were HRV negative, although this difference did not reach statistical significance (median 16 cell $\mathrm{s} / \mathrm{mm}$ basement membrane (IOR 11 to 29) vs median 10 (6.1 to 17.6) for epithelium ( $p=0.056$ ); and median 148 cells $/ \mathrm{mm}^{2}$ (IOR 95 to 217) vs median 95 (36 to 159), for lamina propria $(p=0.076)$ ). There was no association between the total number of APC and the occurrence of HRV.

$\mathrm{CD} \mathrm{a}^{+} \mathrm{DC}$ have previously been found to be significantly increased in the bronchial mucosa of adults with asthma, ${ }^{22}$ but 


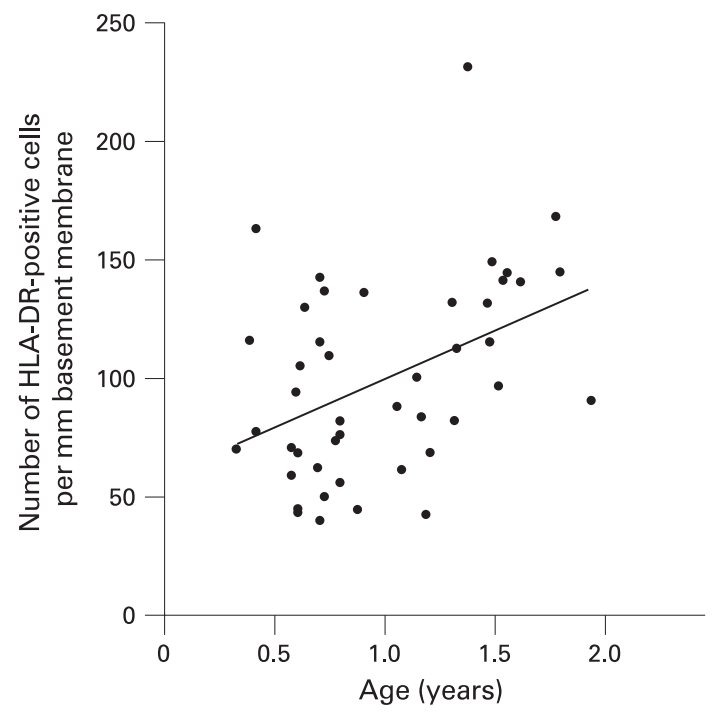

Figure 4 Correlation between the total number of $\mathrm{HLA}^{-\mathrm{DR}^{+}}$cells in bronchial mucosa and age of patients $(r=0.41, p=0.005$, Spearman correlation). Cell numbers are expressed as immunostained cells $/ \mathrm{mm}$ basement membrane, adding the number of intraepithelial cells and the number of lamina propria cells to a depth of $0.25 \mathrm{~mm}$.

we did not find any difference in the distribution of this phenotype among the three clinical groups nor any association with atopy (fig 2, 3B). Nevertheless, when all the patients were considered together, the frequency of $\mathrm{CD}^{+} \mathrm{a}^{+} \mathrm{DC}$ in the epithelial compartment was significantly higher in those reported to have suffered from a respiratory tract infection in the preceding 6 weeks (median 1.6 (IOR 0.4 to 7.7 ) vs 0.3 (0.00 to 3.4 ); $p=0.024$ ) (fig 5), but we found no relation between the number of CD1a ${ }^{+}$DCs and HRV positivity (not shown).

We found no significant associations between any of the cellular phenotypes studied and the levels of serum IgE, eosinophils or serum eosinophil cationic protein (data not shown).

\section{Bronchus associated lymphoid tissue}

Distinct subepithelial aggregates of HLA-DR ${ }^{+}$cells were found in samples from 22 (49\%) of the 45 patients (fig 1B-D). Further immunostaining experiments showed that these cell clusters contained, in addition to APC, $\mathrm{CD} 20^{+} \mathrm{B}$ cells surrounded by $\mathrm{CD}^{+} \mathrm{T}$ cells (fig $1 \mathrm{C}$ ), thereby defining a typical organised BALT structure in the form of an isolated lymphoid follicle (ILF). We observed, however, no sign of germinal centre reactions.

Most of the marker positive cells of ILF were located in the lamina propria, but many $\mathrm{B}$ and $\mathrm{T}$ cells occurred within the overlying epithelium as well. Virtually no B cells were found in the lamina propria outside of the ILF. As noted earlier, most $\mathrm{pDC}$ were situated in the peripheral $\mathrm{T}$ cell containing zones of ILF (fig 1D). Localisation and cellular organisation of these aggregates as typical ILF clearly demonstrated that they represented BALT.

There was no difference in the frequency of ILF among the three patient categories (group A, 7/14; group B, 9/18; and group $C, 6 / 13$ ) and no relation to atopy status (atopic 10/19; non-atopic 12/26), age, HRV positivity or history of respiratory infections (not shown).

\section{Foxp3 ${ }^{+}$regulatory $\mathrm{T}$ cells}

Because Foxp3 is the best current marker to identify Treg, costaining for CD3 and Foxp3 was performed to identify this

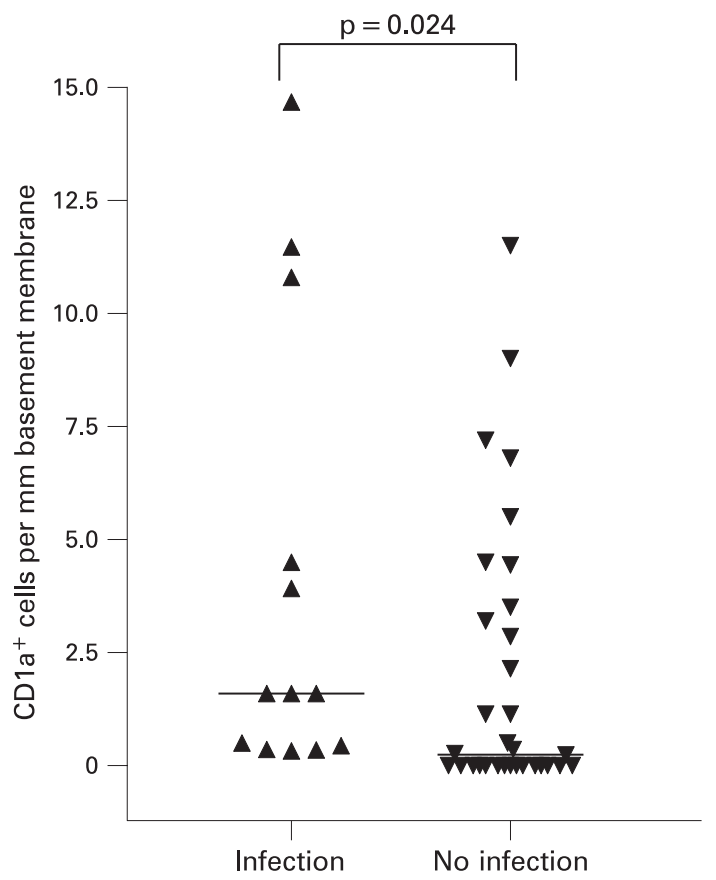

Figure 5 The number of $\mathrm{CD} 1 \mathrm{a}^{+}$cells in bronchial epithelium of children reported to have suffered a respiratory infection in the 6 weeks prior to biopsy was significantly higher than in those without a history of a recent infection ( $p=0.024$, Mann-Whitney test). Horizontal lines represent medians.

phenotype in situ. As there has been some recent controversy concerning the specificity of different anti-Foxp3 antibodies, ${ }^{23}{ }^{24}$ we performed parallel staining experiments with two different mAbs directed against Foxp3 (clone PCH101 and 259D/C7). Both $\mathrm{mAbs}$ gave similar staining results. All examined ILF contained $\mathrm{CD}^{+} \mathrm{Foxp}^{+}$cells (fig 1E) but their distribution was highly compartmentalised and $\mathrm{CD}^{+} \mathrm{Foxp}^{+}$cells appeared only occasionally in lamina propria outside these lymphoid structures (fig 1F). There was no association between the occurrence of CD3+Foxp3 ${ }^{+}$cells and age, lung function, atopy, history of respiratory infections or any of the other clinical parameters (not shown).

\section{DISCUSSION}

This study showed that a network of strongly HLA-DR ${ }^{+}$cells can be established in human bronchial mucosa shortly after birth. Tschernig and colleagues ${ }^{25}$ previously observed in autopsy material from infants who had died from trauma or sudden infant death syndrome (SIDS) that HLA-DR ${ }^{+}$cells were not regularly found in human tracheal mucosa within the first year of life. This finding suggested that the influx of APC into the airway mucosa is a very slow process during infancy. However, more recently the same group reported from SIDS cases that the tracheal mucosa contained a relatively high number of HLA$\mathrm{DR}^{+}$cells, similar to that found here $\left(200-300 \mathrm{cells} / \mathrm{mm}^{2}\right.$ of lamina propria). ${ }^{26}$ The striking difference between their two studies might be explained by the fact that the first one was performed on formalin fixed and paraffin embedded tissue whereas the latter was performed on frozen sections. Formalin cross links proteins, thereby masking antigenic sites in tissue specimens. By contrast to our approach, Tschernig and colleagues $^{25}$ did not perform epitope retrieval of formalin fixed tissue. Such pretreatment is necessary to break cross links between proteins to unmask hidden epitopes. This procedure 
often has a dramatic effect on the immunohistochemical staining intensity, which most likely explained why we found relatively high numbers of HLA-DR+ cells.

For obvious ethical reasons we were unable to obtain biopsy specimens from healthy control children. However, the latter study by Tschernig and colleagues ${ }^{26}$ suggested that the density of APC found by us could in fact reflect the normal situation within this age group. Although respiratory infections have been suggested to contribute to the pathogenesis of SIDS, many studies have failed to document overt signs of mucosal inflammation in the victims. ${ }^{27}{ }^{28}$ Also, both our study and that of Tschernig and colleagues demonstrate that the density and distribution of resident APC in the conducting airways of infants were similar to that observed in the nasal mucosa of healthy adults. ${ }^{7}$ In both the upper and lower airway mucosa there was a dominance of macrophages. We detected very few $\mathrm{pDC}$, except in BALT structures, so the DC population consisted mainly of the myeloid subtype.

The biopsy specimens used in our study were all obtained from the carina. This anatomical region represents part of the central airways and animal studies have shown that accumulation of immune cells in this area correlates with disease manifestations in experimental models of respiratory allergy and infections. We therefore believe that our material is representative of the airway mucosa; but it cannot be ruled out that cellular infiltrates further down the bronchial tree might differ from those near the carina, which could have affected the observed clinical phenotype.

Together, the findings discussed above suggest that the distribution and relative proportions of different APC subsets are established quite early in life. The results furthermore imply that any defect in respiratory immunity in early childhood might not be due to limited migratory capacity of circulating APC subtypes, but could rather be ascribed to impaired ability of resident airway APC to deliver adequate stimulatory signals to $\mathrm{T}$ cells.

A finding that merits specific comment is the relatively high frequency of organised BALT structures identified as ILF in bronchial mucosa at this early age. The ILF were defined as aggregated $\mathrm{HLA}-\mathrm{DR}^{+} \mathrm{APC}$ together with distinct $\mathrm{B}$ and $\mathrm{T}$ cell areas. After childhood, the frequency of BALT structures reportedly decreases with age, and controversy exists concerning whether they represent a constitutive part of normal adult airways. ${ }^{29-31}$ Tschernig and Pabst ${ }^{32}$ did not find ILF in normal autopsy lungs of adults and only in $40 \%$ of healthy adolescents and children. The frequency was similar in SIDS victims and victims of traumatic death. ${ }^{33}$ We found bronchial ILF in $\sim 50 \%$ of the biopsy specimens. Taking into account the small size of these samples, we believe that our result is an underestimate and that such BALT structures may represent a general feature in the lower airways at this early age. The function of BALT is not well understood. Recent data from mouse experiments have demonstrated that such structures can be induced and generate strong primary $B$ and $T$ cell responses to inhaled influenza virus. ${ }^{34}$ There is also evidence to suggest that human BALT is inducible. ${ }^{35}$ We found that the bronchial ILF contained Foxp3 ${ }^{+} \mathrm{T}$ cells, presumably Treg, and some pDC in the surrounding T cell zones. This is consistent with the notion that $\mathrm{pDC}$ primarily traffic organised lymphoid tissue. ${ }^{36}$ Interestingly, experiments in mice recently suggested that pulmonary $\mathrm{pDC}$ are crucial for maintenance of airway tolerance to inhaled allergens, ${ }^{37}$ probably through their generation of Treg. In vitro studies have likewise shown that $\mathrm{pDC}$ can induce $\mathrm{T}$ cells with suppressive functions, further emphasising a tolerogenic potential for these cells. ${ }^{12}{ }^{38}$
The finding that both Foxp3 $3^{+}$Treg and $\mathrm{pDC}$ are present in bronchial ILF implies that these BALT structures may be instrumental in maintaining mucosal homeostasis. ${ }^{39}$

We noted that a fraction of intraepithelial HLA-DR ${ }^{+}$cells extended projections between epithelial cells into the airway lumen. We have recently reported similar findings in the tracheal mucosa of rats, ${ }^{40}$ and an important role for transepithelial DC in handling luminal antigens has been demonstrated in the gut of mice. ${ }^{4142}$ This is the first study to demonstrate the existence of such "snorkelling" DC in the airway mucosa of humans. The functional implications of this finding remains to be determined, but it is tempting to speculate that these DC continuously sample luminal antigens, which would enable them to rapidly alarm the regional adaptive immune system by capturing pathogens prior to invasion. Conversely, in the absence of danger signals, these migrating and antigen carrying DC may deliver tolerogenic signals to cognate $\mathrm{T}$ cells in the draining lymph nodes. ${ }^{43}$ Such functional possibilities (in addition to the presence of BALT structures) might explain how immune surveillance and tolerance induction could operate in human bronchial mucosa.

All of the patients studied here had clinical persistent airway symptoms and were divided into three groups based on lung function measurements assessed by plethysmography. We detected no difference in bronchial densities of APC subsets in relation to clinical classification, which suggested that factors other than the mucosal density of these cells in the mucosa are responsible for the differences in clinical phenotype. Infants with wheezing and impaired lung function represent a heterogeneous group and the diagnosis of bronchial asthma can only be made at a later stage. ${ }^{44}$ Notably, in our patients, neither reticular basement membrane thickening nor signs of eosinophil airway inflammation were observed, ${ }^{18}$ which emphasises the fact that these young children have not yet developed the classical morphological and immunopathological features of bronchial asthma. Thus the possible significance of our in situ immunophenotyping in relation to persistent airway disease will only become evident on follow-up of the same patients.

Acknowledgements: We wish to thank nurses Tuija Rito and Helena Punkari for their skill and care with the infants, and especially the children themselves and their parents.

Funding: Nummela Sanatorium Foundation, Paediatric Research Foundation, Helsinki University Central Hospital Research Fund, Sigrid Juselius Foundation, Liv och Hälsa Foundation, AstraZeneca Finland, University of Oslo and the Norwegian Asthma and Allergy Foundation

Competing interests: None.

Ethics approval: The study was approved by the local ethics committee.

\section{REFERENCES}

1. Holt PG, Upham JW, Sly PD. Contemporaneous maturation of immunologic and respiratory functions during early childhood: implications for development of asthma prevention strategies. J Allergy Clin Immunol 2005;116:16-24.

2. Marchant A, Goldman M. T cell-mediated immune responses in human newborns: ready to learn? Clin Exp Immunol 2005;141:10-18.

3. Holt PG. Programming for responsiveness to environmental antigens that trigger allergic respiratory disease in adulthood is initiated during the perinatal period. Environ Health Perspect 1998;106(Suppl 3):795-800.

4. Upham JW, Lee PT, Holt BJ, et al. Development of interleukin-12-producing capacity throughout childhood. Infect Immun 2002;70:6583-8.

5. Banchereau J, Steinman RM. Dendritic cells and the control of immunity. Nature 1998;392:245-52.

6. Hunt DW, Huppertz HI, Jiang HJ, et al. Studies of human cord blood dendritic cells: evidence for functional immaturity. Blood 1994;84:4333-43.

7. Jahnsen FL, Gran E, Haye R, et al. Human nasal mucosa contains antigen-presenting cells of strikingly different functional phenotypes. Am J Respir Cell Mol Biol 2004;30:31-7. 
8. Holt PG, Haining S, Nelson DJ, et al. Origin and steady-state turnover of class II $\mathrm{MHC}$-bearing dendritic cells in the epithelium of the conducting airways. $J$ Immunol 1994;153:256-61.

9. Holt PG, Stumbles PA, McWilliam AS. Functional studies on dendritic cells in the respiratory tract and related mucosal tissues. J Leukoc Biol 1999;66:272-5.

10. Nelson DJ, Holt PG. Defective regional immunity in the respiratory tract of neonates is attributable to hyporesponsiveness of local dendritic cells to activation signals. J Immunol 1995;155:3517-24.

11. Kvale EO, Floisand Y, Lund-Johansen F, et al. Plasmacytoid DCs regulate recall responses by rapid induction of IL-10 in memory T cells. Blood 2007;109:3369-76.

12. Ito $\mathbf{T}$, Yang $\mathrm{M}$, Wang $\mathrm{YH}$, et al. Plasmacytoid dendritic cells prime IL-10-producing $T$ regulatory cells by inducible costimulator ligand. J Exp Med 2007:204:105-15.

13. Borras FE, Matthews NC, Lowdell MW, et al. Identification of both myeloid CD11c+ and lymphoid CD11c- dendritic cell subsets in cord blood. Br J Haematol 2001:113:925-31.

14. Sakaguchi S. Naturally arising CD4+ regulatory T cells for immunologic selftolerance and negative control of immune responses. Annu Rev Immunol 2004;22:531-62.

15. Sakaguchi S, Ono M, Setoguchi R, et al. Foxp3+CD25+CD4+ natural regulatory T cells in dominant self-tolerance and autoimmune disease. Immunol Rev 2006:212:8-27.

16. Umetsu DT, Dekruyff RH. The regulation of allergy and asthma. Immunol Rev 2006;212:238-55.

17. Michaelsson J, Mold JE, McCune JM, et al. Regulation of T cell responses in the developing human fetus. J Immunol 2006;176:5741-8.

18. Saglani S, Malmstrom K, Pelkonen AS, et al. Airway remodeling and inflammation in symptomatic infants with reversible airflow obstruction. Am J Respir Crit Care Med 2005; 171:722-7.

19. Lindahl H, Rintala R, Malinen L, et al. Bronchoscopy during the first month of life J Pediatr Surg 1992;27:548-50.

20. Malmstrom K, Pitkaranta A, Carpen 0, et al. Human rhinovirus in bronchia epithelium of infants with recurrent respiratory symptoms. J Allergy Clin Immunol 2006:118:591-6.

21. Soler $\mathbf{P}$, Moreau A, Basset F, et al. Cigarette smoking-induced changes in the number and differentiated state of pulmonary dendritic cells/Langerhans cells. Am Rev Respir Dis 1989;139:1112-17.

22. Moller GM, Overbeek SE, Van Helden-Meeuwsen CG, et al. Increased numbers of dendritic cells in the bronchial mucosa of atopic asthmatic patients: downregulation by inhaled corticosteroids. Clin Exp Allergy 1996;26:517-24.

23. Tran DQ, Ramsey H, Shevach EM. Induction of FOXP3 expression in naive human CD4+FOXP3 T cells by T-cell receptor stimulation is transforming growth factor\{beta\} dependent but does not confer a regulatory phenotype. Blood 2007; 110:2983-90

24. Pillai V, Karandikar NJ. Attack on the clones? Human FOXP3 detection by PCH101, 236A/E7, 206D, and 259D reveals 259D as the outlier with lower sensitivity. Blood 2008;111:463-4.

25. Tschernig T, Debertin AS, Paulsen F, et al. Dendritic cells in the mucosa of the human trachea are not regularly found in the first year of life. Thorax 2001;56:427-31.

26. Tschernig T, De Vries VC, Debertin AS, et al. Density of dendritic cells in the human tracheal mucosa is age dependent and site specific. Thorax 2006;61:986-91.
27. Debertin AS, Tschernig T, Schurmann A, et al. Coincidence of different structures of mucosa-associated lymphoid tissue (MALT) in the respiratory tract of children: no indications for enhanced mucosal immunostimulation in sudden infant death syndrome (SIDS). Clin Exp Immunol 2006;146:54-9.

28. Hiller AS, Kracke A, Tschernig T, et al. Comparison of the immunohistology of mucosa-associated lymphoid tissue in the larynx and lungs in cases of sudden infant death and controls. Int J Legal Med 1997;110:316-22.

29. Pabst R. Is BALT a major component of the human lung immune system? Immunol Today 1992;13:119-22.

30. Hiller AS, Tschernig T, Kleemann WJ, et al. Bronchus-associated lymphoid tissue (BALT) and larynx-associated lymphoid tissue (LALT) are found at different frequencies in children, adolescents and adults. Scand J Immunol 1998;47:159-62.

31. Sue-Chu M, Karjalainen E, Altraja A, et al. Lymphoid aggregates in endobronchial biopsies from young elite cross-country skiers. Am J Respir Crit Care Med 1998; 158:597-601

32. Tschernig T, Pabst R. Bronchus-associated lymphoid tissue (BALT) is not present in the normal adult lung but in different diseases. Pathobiology 2000;68:1-8.

33. Tschernig T, Kleemann WJ, Pabst R. Bronchus-associated lymphoid tissue (BALT) in the lungs of children who had died from sudden infant death syndrome and other causes. Thorax 1995;50:658-60.

34. Moyron-Quiroz JE, Rangel-Moreno J, Kusser K, et al. Role of inducible bronchus associated lymphoid tissue (iBALT) in respiratory immunity. Nat Med 2004;10:927-34.

35. Rangel-Moreno J, Hartson L, Navarro C, et al. Inducible bronchus-associated lymphoid tissue (BALT) in patients with pulmonary complications of rheumatoid arthritis. J Clin Invest 2006;116:3183-94.

36. Liu YJ. IPC: professional type 1 interferon-producing cells and plasmacytoid dendritic cell precursors. Annu Rev Immunol 2005;23:275-306.

37. de Heer HJ, Hammad H, Soullie T, et al. Essential role of lung plasmacytoid dendritic cells in preventing asthmatic reactions to harmless inhaled antigen. J Exp Med 2004;200:89-98

38. Moseman EA, Liang $X$, Dawson AJ, et al. Human plasmacytoid dendritic cells activated by $\mathrm{CpG}$ oligodeoxynucleotides induce the generation of CD4+CD25+ regulatory T cells. J Immunol 2004;173:4433-42.

39. Gilliet M, Liu YJ. Human plasmacytoid-derived dendritic cells and the induction of $T$ regulatory cells. Hum Immunol 2002;63:1149-55.

40. Jahnsen FL, Strickland DH, Thomas JA, et al. Accelerated antigen sampling and transport by airway mucosal dendritic cells following inhalation of a bacterial stimulus. J Immunol 2006;177:5861-7.

41. Chieppa M, Rescigno M, Huang AYC, et al. Dynamic imaging of dendritic cell extension into the small bowel lumen in response to epithelial cell TLR engagement. J Exp Med 2006;203:2841-52

42. Rescigno $\mathbf{M}$, Urbano $\mathbf{M}$, Valzasina $\mathrm{B}$, et al. Dendritic cells express tight junction proteins and penetrate gut epithelial monolayers to sample bacteria. Nat Immunol 2001:2:361-7.

43. Steinman RM, Hawiger D, Liu $\mathrm{K}$, et al. Dendritic cell function in vivo during the steady state: a role in peripheral tolerance. Ann N Y Acad Sci 2003;987:15-25.

44. Martinez FD. Development of wheezing disorders and asthma in preschool children 1. Pediatrics 2002;109:362-7.

No busy professional has time to browse through all pertinent journals to find relevant articles, but with Related Collections you no longer have to. Follow the "Related Collections" link from any article and use the "Show Collections from other Journals" to expand your search across all BMJ Journals. Or simply follow the "Browse by topic" link on the home page. By setting up your own collections and receiving email alerts every time an article is added to your chosen area, you can build up your own significant body of knowledge. 